be borne in mind. As is to be expected, the prognosis is extremely bad, but, in some cases, there is only diminution of fibrinogen, without total absence, and then the prognosis is fairly good if the patient leads a careful life. Transfusion is a life-saving measure, but its effects are, of course, transient.

Differentiation from haemophilia is simple. The addition of a few drops of the thromboplastin solution used in the estimation of prothrombintime causes prompt coagulation of haemophilic blood, but not of that in afibrinogenaemia.

Again, the family history is significant, especially as consanguinity of the parents is almost invariable in the cases of congenitally total absence of fibrinogen.

More striking and certainly commoner are cases of acquired absence or diminution of fibrinogen. In these there is a history of severe bleeding, perhaps after tooth extraction on one occasion, and medical advice is sought because of apprehension about subsequent operations. Often, at the time of examination, the fibrinogen content of the plasma is found to be normal because, fortunately, acquired afibrinogenaemia is almost invariably a transient state, which can be detected only at the time of the initial bleeding or, by chance, if a very complete blood examination is performed for some other reason. Relapses occur but are by no means common.

Confusion between such an acquired condition and thrombocytopenic purpura is not possible if the platelets are enumerated; the only other abnormality in the blood in these cases is, possibly, anaemia due to haemorrhage. Nevertheless, the history in the two maladies may be confusing, because spontaneous remissions occur in both.

Among the confused chapters in haematology, purpura is the most confused, and although the present paper has presented some practical points it does not claim to have done anything to lighten the darkness that covers the more recondite aspects of the subject.

\section{REFERENCES.}

ALTSCHULE, M. D., New Engl. Journ. Med., I933, 227, 471 . BENDA, R., Ann. Med., I930, 27, 190.

\title{
A TECHNIQUE OF LOCAL ANAESTHESIA FOR ABDOMINAL OPERATIONS
}

\author{
By Harold Dodd, CH.M., F.R.C.S.(ENG.). \\ Surgeon: Royal Hospital, Richmond; King George Hospital, Ilford; Princess Louise Hospital, Kensington. \\ Assistant Surgeon: London Homoeopathic Hospital.
}

Preamble.-The purpose of this paper is to outline a method of local anaesthesia for operations on the abdomen. It has been used since June I937, in over two thousand procedures. It was at first used as an alternative anaesthetic to general or spinal for abdominal operations, and in this way its qualities became well known. It is offered now as forming the principle partner of a combination of anaesthetics, i.e., local anaesthesia plus a sleep-producing agent. In I 942 one of my anaesthetic colleagues called the combination "Balanced Anaesthesia" and it has proved to be a singularly satisfactory method. One housesurgeon said, "Every partial gastrectomy got a chest of some sort after a general or spinal anaesthetic, but not with a local." Actually, whilst using local anaesthesia, I have lost one patient by death upon the table, but a considerable number in the same time when spinal and general anaesthesia was being used. Further, the post-operative morbidity and mortality is less. Patients, according to the opinion of experienced ward sisters, have a better post-operative convalescence.

Balanced Anaesthesia.-Balanced Anaesthesia for abdominal operations enables a patient's needs to be fully met, whilst satisfying the anaesthetist, surgeon, and surgical team. The local consists of a simple readily-acquired technique (anaesthetists and house-surgeons quickly learn it) supplemented by omnopon and scopolamine, open ether or continuous pentothal to produce sleep. When carefully injected it gives complete relaxation for major procedures and easy closure of the wound. It permits successful major operations on the poorrisk and elderly patient, especially on the upper abdomen, without heaving jerky, eviscerating respirations. The consistent quality of the anaesthesia with the quiet breathing and slow bleeding contrasts with general anaesthetics which are characterised by variability from perfection to exasperation for the surgeon and anaesthetist. In my practice Balanced Anaesthesia has abolished acrimonious incidents with the anaesthetist and has consolidated friendships. The local anaesthetic lasts for two or three hours, there is no need to hurry an operation because of the increasing dose of anaesthetic. The time needed for painstaking craftmanship can be given. The amount 
of general anaesthetic to maintain sleep is small and it is often stopped after the manipulative part of the operation is done and before closure.

The supplementary general anaesthetic is necessary because insensitivity sufficient for a complete abdominal exploration satisfactory alike to patient and surgeon has not so far been achieved by the local anaesthetic plan to be outlined. The occasional moans and movement by a conscious patient operated on by local anaesthesia alone upset those working in the theatre.

\section{Objections to Local Anaesthesia}

I. Time.-Local Anaesthesia is unsuitable for those surgeons who have long operating lists and who are constrained to compete with the clock. Five minutes is required to insert the local. With practice and organisation the time can be halved. The full relaxation makes each step comfortable and expeditious.

2. Obscured Anatomy.-Some surgeons say that the oedema of the anaesthetic obscures the anatomy. This may be so, immediately after the injection, but I have not experienced it. On the contrary the injection shows the tissue planes and facilitates dissection, e.g. in cholecystectomy.

3. The Additional Burden on the Surgeon.The administration of the local anaesthetic is an extra duty on the surgeon. The parietes can be infiltrated by the anaesthetist, but, the viscera are done when the moment arrives by the operator, which adds a few minutes to the procedure. The surgeon may be physically too tired to insert the anaesthetic, especially if he has a big list.

4. Gentleness.-Operating with local anaesthesia requires gentle precise work with a scalpel; a criterion for the best surgery under any anaesthetic.

The Patient's Comfort.-The patient's peace of mind and physical comfort is the surgeon's responsibility. Lying on the hard operating-table, even with a sorbo mattress is soon unpleasant. Body ease and the operation are assisted by an extra pillow under the head and one beneath the knees, and they also relax the abdominal muscles by flexing the thorax and pelvis.

Mental tranquillity is obtained by adequate pre-anaesthesia. Standardised pre-medication is uncertain for the effects depend on the age, type, and illness of the patient. Combinations of omnopon and scopolamine work satisfactorily. One plan is, $1 \frac{1}{2}-2$ hours before the operation, Omnopon grs. $\frac{1}{3} \frac{2}{3}$, Scopolamine grs. $\frac{1}{150} \frac{1}{100}$, aiming for patients to be asleep an hour before operation: if not, another omnopon gr. $\frac{1}{3}$ is given. Burge and James (B.M.J., Dec. 27, I94I) recommended omnopon intravenously slowly until the patient is asleep, stipulating that the respirations must not fall below fourteen per minute. I lost a patient from respiratory depression after such a dose.

\section{Supplementary Anaesthesia During the Operation}

I. Open Ether.-A safe and simple sleep-agent is by open ether not deep enough to require a gag, tongue forceps or an air-way. It is emphasised that it is sleep to light anaesthesia. The induction may be by $0.4-0.5$ grms. of pentothal or evipan. The sleep agent is often discontinued when the laparatomy and the parts of the operation needing traction or retraction are done. The local anaesthetic, the pre-anaesthesia and the "hang-over" of the ether or pentothal carry the patient through even large operations.

2. The Light Intravenous Barbiturates.-The intravenous light barbiturates are first-class for local anaesthesia, with the minimum of "going under" discomfort. Two methods are available. The first is the slow injection of $0 \cdot 4-0.5 \mathrm{grms}$. of $2 \cdot 5-5$ per cent pentothal or evipan and the second, the safer, although more difficult technically, is the administration of pentothal to cause sleep, plus "trickle" dose of $\frac{1}{2}$-I c.c. of $2 \cdot 5$ per cent pentothal at a time as the patient requires it. A two-hour operation has repeatedly been performed with $0 \cdot 5-0.7$ grms. of pentothal plus the local.

The danger of cutting down on veins for intravenous drips and injections of pentothal is emphasised. The mortality is, in my practice, at least I per cent from infection and thrombosis, besides frequent septic arms, pain, and incapacitation. I find that this experience is fairly general in Britain.

3. Gas and Oxygen.-As a junior partner for local anaesthesia, gas and oxygen is unsuitable; it causes heaving respiration, muscle tautness and in 50 per cent of patients, post-operative vomiting, often beginning on the operating table and lasting for 6-12 hours.

\section{Local Anaesthetic Drugs}

Three local anaesthetic media are availablethey are, procaine $\frac{1}{2}-I$ per cent. Amethocaine $\frac{1}{1000}$, and nupercaine $\frac{1}{1500}$. A combination is novocaine $\frac{1}{4}$ per cent, and nupercaine $\frac{1}{1400}$ (James and Burge, B.M.J., December 27, I94I). They are sterilised by boiling or autoclaving, although some preparations of procaine stand neither and decompose.

\section{The Characters of the Local Anaesthetic}

\section{Drugs}

Procaine.-(Novocaine) $\frac{1}{2}$ per cent anaesthetises almost immediately for forty to sixty minutes and is non-toxic. 
Amethocaine.-(Decicaine, Pantocaine) $\frac{1}{1000}$ acts in five minutes for two to three hours and is slightly toxic. I have used this preparation since June I93́7.

Nupercaine.-(Percaine) $\frac{1}{1500}$ acts in ten minutes for three to four hours and is fairly toxic.

Adrenalin.-Adrenalin $\frac{1}{1000}$ (from an ampoule) is added to the local anaesthetic after sterilisation, 2 minims per ounce.

Volumes of 200-300 c.cs. of each anaesthetic can be used safely.

Colour of the Anaesthetic.-The anaesthetic is coloured blue with a few drops of indigo-carmine (as used for chromoscopy); it is harmless. It shows where the anaesthetic is, where the tissues are blue there is anaesthetic and, therefore, anaesthesia, where there is no blue there is no anaesthetic and no anaesthesia.

The Apparatus.-The apparatus is as in the diagram and with the exception of the Dunn twoway valve, it is improvisable. Two 5 c.c. syringes used alternately are admirable. The surgeon and anaesthetist or assistant do either side together so reducing the injection time. Syringes larger than Io c.cs: tire the hand.

Needles. - Three types of needles are needed, mainly a No. I5 or I6 hypodermic needle $\frac{7}{8}$ in. long and another similar but $\mathrm{I}+1$ in. in length for very fat patients, and a modern spinal anaesthetic needle (No. I9 or 20) for injection of the splanchnic ganglia and the mesenteries. All are available in hospitals.

Asepsis.-Asepsis of the apparatus, of the anaesthetic, in the operator and towelled operation area is essential. When the injections were made by wet scrubbed hands on an untowelled abdomen, painful indurated areas followed and once or twice an abscess. A mild infection is introduced from the unsterile surroundings. Perfect healing follows sterile injections.

\section{The Administration of the Local Anaesthetic}

The local anaesthetic is administered in two complementary plans: by local infiltration of the actual operation site, plus a regional field-block of the abdominal wall and splanchnic sympathetic plexus and vagus.

Three points are observed. First, that the injection under the skin raises a "pig skin" wheal. The second, that the injection is made continuously as the needle is advanced into the tissues, so that a stream of anaesthetic under pressure precedes the needle point. Last, time is needed for the anaesthetic to act so the operation is arranged so that while the anaesthesia develops a step such as tying-off blood vessels, application of skin cloths, etc., is done.

r. The Local Infiltration (for abdominal operations).- The local infiltration of the incision includes the skin and the full thickness of the abdominal wall. It takes effect while the regional block is made.

Using a 5 c.c. syringe (holds 6 c.cs.) fitted with the short No. I5-I6 needle, 3 c.cs. are placed subcuticularly for the length of the needle, raising the "pig skin" wheal and then almost withdrawing it is re-inserted vertically down, injecting simultaneously thus anaesthetising the fat, rectus sheath, rectus muscle, posterior rectus sheath and peritoneum. The rectus sheath is good for the pooling and spread of the anaesthetic.

2. The Regional Infiltration.-The regional infiltration has been standardised and is readily learned. It infiltrates with 6-7 c.cs. of anaesthetic the spots where the 6th, 7 th, 8th, 9th, Ioth, IIth, and I2th nerves are known to lie.

\section{The Points Are as Follows:-}

The 6th nerve is $\mathrm{I}$ in. along the costal margin from the mid line. The needle is directed vertically down to the under-surface of either costal margin.

The 7th Dorsal Nerve is hit-off by vertical punctures below the costal margin, midway between the middle line (at the ensiform cartilage) and the point of intersection of the linea semilunaris with the costal margin. The injection continues steadily as the needle penetrates to its full length through the anterior rectus sheath, rectus muscle, and posterior rectus sheath to the peritoneum.

The 8th and 9th nerves are located on either side of the linea semi-lunaris, at the costal margin. The abdominal wall is thin here so that the needle is advanced slowly at $45^{\circ}$ to the surface. The skin penetrated, the needle is inclined upwards and inwards to the deep costal margin through the outer edge of the rectus muscle. Then the needle is almost withdrawn and is re-directed downwards and outwards along the costal edge, approximately at $45^{\circ}$, so that the aponeurosis of external oblique, internal oblique, and transversalis are infiltrated just outside the linea semilunaris.

The "arrow-head" injection-Ioth, IIth, and I2th nerves are treated through a single puncture which is made in the mid-axillary line exactly opposite to the umbilicus. It is called the "arrowhead" injection, and is an invaluable instillation for upper and lower abdominal procedures. Each injection infiltrates evenly the six structures to the peritoneal cavity, i.e. the external and internal oblique muscles, transversalis muscle and fascia, extra peritoneal tissue and peritoneum. In fat 
and muscular subjects care is needed to penetrate all the layers.

The IIth Nerve.-The first insertion, in the midaxillary line opposite the umbilicus, passes in horizontally, this is important, for there is a tendency to direct it obliquely forwards and so miss the deeper structures, which gives a tight retracting peritoneum. The muscles here are thick and there is no risk of penetrating the peritoneum because it is very mobile. The needle is ultimately thrust in hard to the shoulder injecting continuously. The needle is now almost withdrawn.

The Ioth Nerve.-The syringe is re-charged and the second injection is directed upwards and inwards along and in line with the costal cartilage towards the tip of the ensiform cartilage, this blocks the Ioth nerve, care is taken not to break or bend the needle by vigorous contact with the cartilaginous costal margin.

The I2th Nerve.-This is blocked after almost withdrawing the needle, and re-directing it horizontally downwards and inwards at the symphysis pubis. Again the needle is pressed in cautiously lest it is snapped off by impact with the crest of the ilium.

Using 7-Io c.cs. for each nerve, it needs up to roo c.cs. to deal with 6-12th nerves on each side, i.e. for the regional block.

The Danger of Perforating the Bowel.-Is there any danger of puncturing the bowel wall? Using a fine short hypodermic needle and injecting continuously as the needle is advanced, it is unlikely that the peritoneum will be penetrated, still less the bowel punctured. The peritoneum is fairly mobile in the central abdomen and considerably so at the periphery and it is raised by the stream of anaesthetic under pressure which precedes the needle point. Slow injections are necessary because rapid distension of the tissues causes pain and the peritoneum may be punctured. I have occasionally seen an area of bowel wall coloured blue, indicating penetration of the peritoneum and contact with the bowel. No trouble has followed it. Casual work, trusting to the Sleep to fill the deficiencies of the injections, gives unsatisfying results. The local anaesthetic by its consistent effectiveness is the senior partner of the anaesthesia.

\section{The Operation}

The incision is made to the parietal peritoneum when the local infiltration and regional field-block are done.

Parietal Peritoneum.-The parietal peritoneum is extremely sensitive, and it is essential to anaesthetise it separately. It is performed before the peritoneum is opened by inserting the fine needle just into its surface and raising wheals as with the skin. Bending the needle helps the injection. The peritoneum becomes thickened to $\frac{1}{4}$ to $\frac{1}{2}$ inch. An alternative method is to do it from the deep side of the peritoneum after it has been opened, but the former is probably the best. The vessels of the wound are tied off while it takes effect. I recall a patient, when local anaesthesia was used solely, in whom the infiltration of the peritoneum was omitted. During closing, although he was relaxed, each time the peritoneum was grasped by the forceps, and penetrated by the needle, he groaned.

\section{Comments on Various Abdominal Incisions}

I. The Middle-Line Incision.-Middle-line incisions are made quickly and easily closed. In my experience they are prone to burst and up to 20 per cent herniate later. They are "popular" in Britain now. Local anaesthesia works admirably for it. The round ligament is first wiped over to the right, before opening the peritoneum the upper edges of the latter are inclined to bleed.

2. The Right and Left Para-Median Incision.The right and left para-median incisions for upper abdominal operations begin at the tip of the ensiform cartilage, curve out along the costal margin for one inch and then run down $\frac{1}{2}$ inch from and parallel to the middle line as far as necessary, usually to the umbilicus. This is infiltrated superficially and vertically, and the regional anaesthetic is inserted, the surgeon and assistant doing their respective sides. This incision allows complete abdominal exploration and most major operations with comfort. It seldom bursts or herniates. The higher up in the abdomen that access is required, e.g. the cardio-oesophagial junction, the lower down must it extend below the umbilicus in order to permit retraction, this is in addition to it beginning at the ensiform.

3. The Kocher Incision. - The Kocher sub-costal incision is made oftener on the right for biliary operations, occasionally on the left. It begins in the mid-line over the lower portion of the sternum. It splits the linea alba for half an inch and turns downwards and outwards half an inch below and parallel to the right costal margin to half an inch outside the linea semi-lunaris. The skin, fat anterior rectus sheath, transversalis muscle and fascia and peritoneum are divided in the line of the incision. The local infiltration is conducted like that of the mid-line and para-median incisions, that is 3 c.cs. of anaesthetic subcuticularly for the length of the needle and another 3 c.cs. vertically down to the peritoneum.

The regional anaesthetic including the "arrowhead" injection is made, but that into the 6th, 


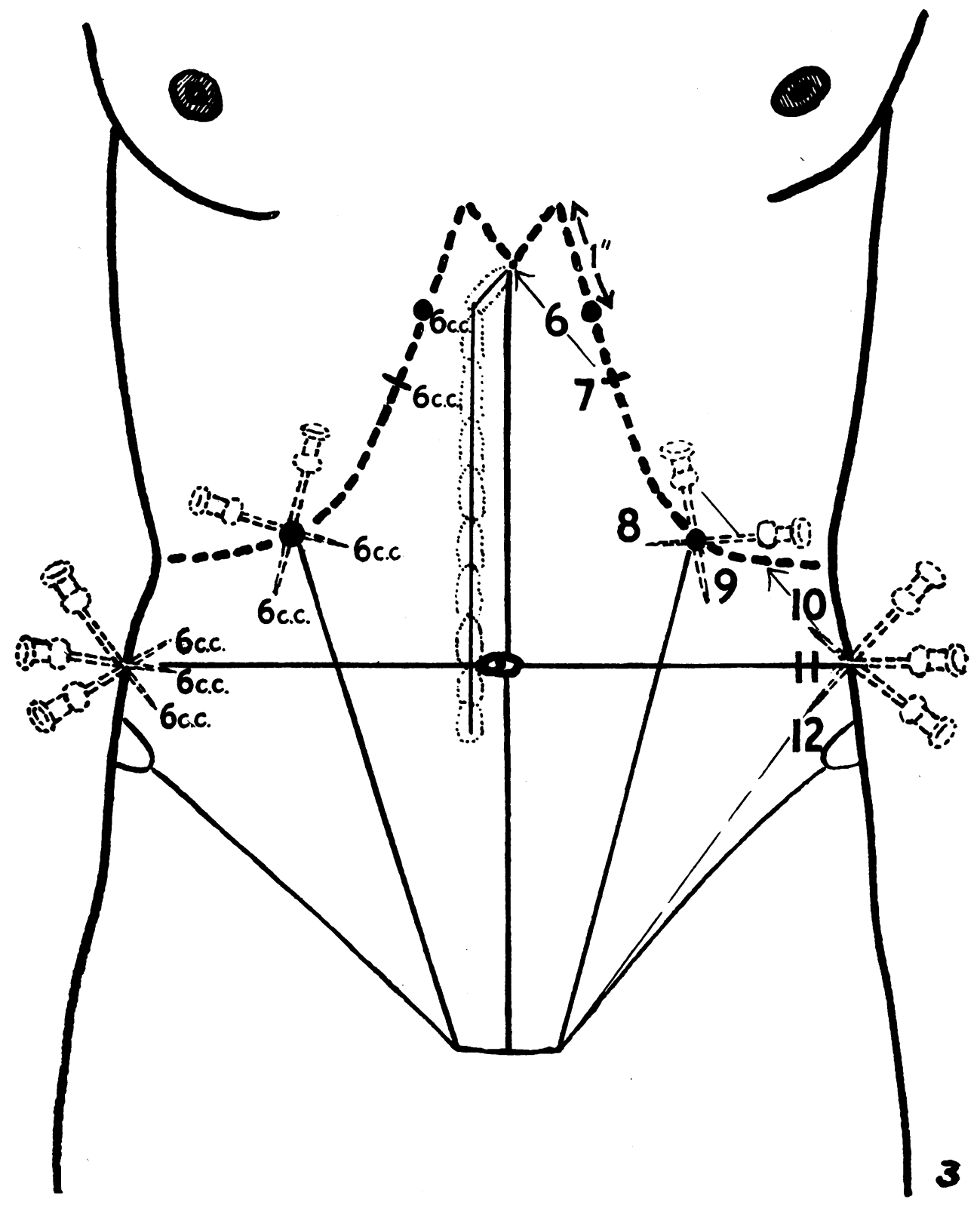

Fig. I.-Local Anaesthesia for Gall-Bladder and Stomach. 


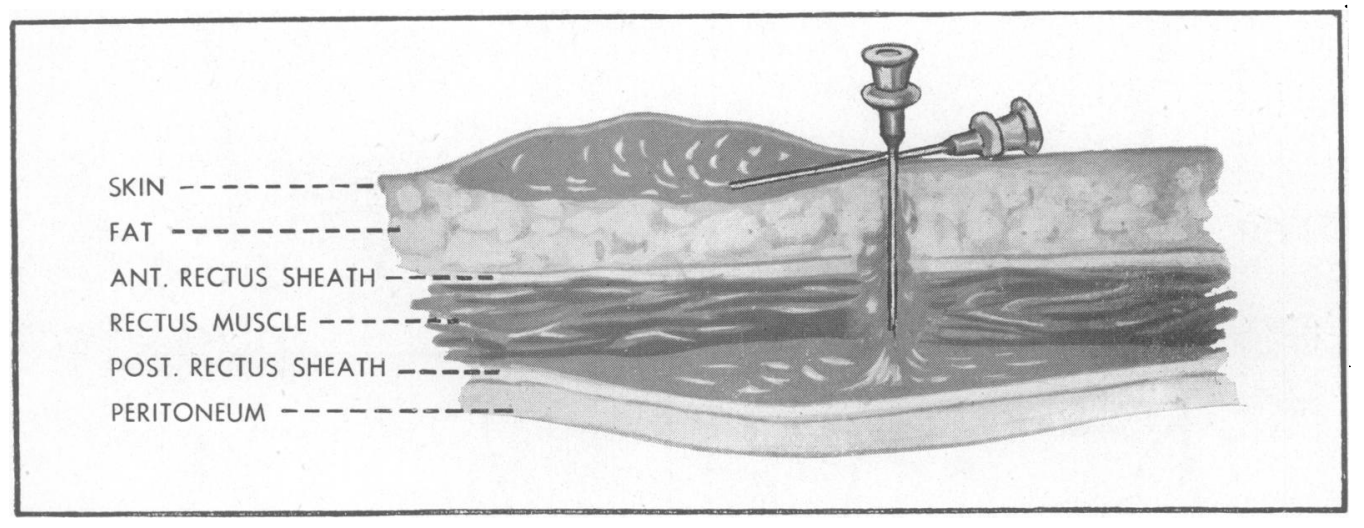


$7_{\text {th, }} 8$ th, and 9 th nerves on the side of the incision is unnecessary being made already by the local infiltration. The transversalis muscle (of varying thickness in patients) with the peritoneum underneath is anaesthetised separately when it is exposed.

4. The Rutherford Morrison Transverse Incision. -T.he Rutherford Morrison Transverse Incision extends across the abdomen from the costal margin to costal margin beginning and finishing at the linea semi-lunaris and curving downwards symmetrically to the middle line, parallel to the abdominal creases and to the nerves of the abdominal wall. Thus there is little danger of injury to the nerves. The skin, fat, rectus muscle and sheath and peritoneum are all divided in this line. The local infiltration is sub-cuticular and full thickness of the abdominal wall over the line of the incision. The regional infiltration is into the costal margins and "arrow-head" injection into the flanks.

It is useful in patients whose inter-costal angle is ninety degrees or over, as for exploration of the biliary tract, duodenum stomach and par excellence, for splenectomy.

5. The Split Muscle (Gridiron Incision) in the Iliac Fossa, also the Incised Iliac Incision.-The essential for this incision is the correct land-mark. It is made at right angles to the line joining the umbilicus to the anterior superior line spine and is centred at the junction of the outer and middle thirds of it. The external oblique, internal oblique, transversalis muscle, and fascia are split in the line of their fibres thus exposing peritoneum.

The Incised Iliac Incision.-An alternative incision to the gridiron or split muscle incisions is that of incising all the muscles in the same line as the skin incision. As it is parallel to the nerves these are seldom divided and a strong scar results.

Extension.-These incisions can be extended either upwards curving outwards to the point of the "arrow-head" injection or downwards to the pubic spine. Superiorly and inferiorly the tissues are divided in the line of the extensions.

The regional field-block is the "arrow-head" injection for the affected side only, and the direct infiltration is from the point of the "arrow-head" injection along the line of the incision to the pubic spine. As in all abdominal incisions, the peritoneum is individually injected before opening the abdomen.

For the appendix, the caecum, the ileo-caecal angle and the meso-appendix are gently lifted out of the abdomen and the last two are injected with 5-Io c.cs. of anaesthetic.

Access to a difficult appendix is achieved by enlarging the incision up or down generously and not by pulling or retracting the wound or groping with the finger. The abdominal wall is unharmed and difficult adherent appendices are robbed of all their anxieties.

6. The Incision for Left Inguinal Colostomy.This incision is made on the left side in precisely the same way as that for the appendix on the right side. It can be likewise extended up and down for laparotomy and also for the abdominal portion of the abdomino-perineal resection of the rectum. It also gives an excellent approach to the ureter in its lower abdominal and pelvic portions.

Infiltration for Supra Pubic and Infra Umbilical Incisions.-I. Regional infiltration is the "arrowhead" incisions into both sides.

The local infiltration is the superficial and vertical instillations in the line of the incision and over a slightly larger area than the opening is expected to be.

\section{Intra-Abdominal Injections of Local Anaesthetic}

I. Regional Insertions.

(a) The Anterior Splanchnic Block.-Incisions in the upper abdomen give easy access to the splanchnic sympathetic nerves and coeliac ganglia lying between and around the aorta and the inferior vena cava on the first lumbar vertebra immediately above the upper border of the pancreas. This is the anterior approach of Braun. The liver is elevated with a suitable retractor (see picture), the stomach is drawn downwards with the left hand to reveal the upper border of the pancreas, and above this the branches of the coeliac axis shining through the gastro hepatic omentum and peritoneum of the posterior abdominal wall. The needle (a spinal needle or Ogilvie pattern) is entered through the peritoneum of the posterior abdominal wall just above the pancreas in the middle line immediately to the right of the abdominal aorta where 40-50 c.cs. of anaesthetic are slowly pooled around the vessels and the associated coeliac ganglia, splanchnic nerves, and it tracks along their branches. The danger of puncture of the aorta or its branches or of the inferior vena cava, is avoided by the tip of the left index finger pressing the aorta outwards to the left and the needle is inserted to right of this point. Preliminary suction with the syringe is made. If blood is withdrawn the injection is stopped, pressure is applied for two minutes and the needle is reinserted. A vessel is perforated very rarely.

(b) The Vagal Block.-To cut off all visceral sensations, especially nausea, retching, and vomiting, the vagus is blocked. The vagus enters through the oesophageal orifice to the anterior and posterior surfaces of the lesser curvature of the stomach. The anterior splanchnic infiltration may track along the coronary (or left gastric) 
artery to the upper lesser curvature and may anaesthetise the vagi, but it is advisable to deliberately infiltrate them by adjustment of the retractors, drawing the body of the stomach downwards and to the right, to expose the lesser curvature at its highest point, and to inject ro c.cs. to the right of the cardiac orifice sub-peritoneally with the long needle. The anaesthetic spreads around the front and posterior surfaces of the lesser curvature and envelopes the vagi.

The regional block for an upper abdominal procedure is now complete, and while it takes effect, the wound vessels are tied off and wound edges are protected by cloths. The anaesthetist deepens the general anaesthetic somewhat in readiness for the abdominal exploration to follow.

(c) Mid Abdomen.-The Small Intestine and Ascending Transverse and Descending Colon.-Their mesenteries are sensitive to light traction and when their blood vessels are clipped. They are anaesthetised regionally and locally by inserting $30-40$ c.cs. of anaesthetic around the origin of the superior mesenteric artery. The transverse colon and omentum are drawn gently out of the abdomen and turned upwards. The origin of the mesentery is revealed by drawing the upper coils of small intestine downwards by the surgeon's left hand. The superior mesenteric artery is in the root of the mesentery and arises in the middle line at the point of divergence of the meso-colon and mesentery. The vessel can be palpated in the free edge of the mesentery, it curves downwards and to the left. The needle is inserted sub-peritoneally, aspirating first to ensure that the point is not in the artery or vein and $30-40$ c.cs. of anaesthetic is introduced and it will be seen to track along and around the right, middle, and left colic arteries and superior mesenteric vessels with their sympathetic nerves.

(d) Lower Abdomen. Operations on the Sigmoid Colon and Upper Rectum.-These procedures are assisted by the Trendelenberg position and packing the small intestine into the upper abdomen. The sigmoid colon and upper rectum is locally and regionally anaesthetised by picking up between the left finger and thumb the root of the sigmoid meso-colon just to the left of the bifurcation of the aorta; this contains the inferior mesenteric vessels and sympathetic plexus. It is injected with 20-30 c.cs. of $0 \cdot I$ per cent amethocaine. The supra-pubic para-median incision must extend well above the umbilicus to give comfortable exposure of this, it is higher than is generally thought. It can also be done through an extended left iliac incision.

The Pelvic Sympathetic Plexuses.-To block the rectum, and reproductory organs and the base of the bladder, the pre-sacral and hypo-gastric sympathetic plexuses must be insulated as well as the inferior mesenteric plexus. It is performed by pooling $20-30$ c.cs. of amethocaine $I$ in $I, 000$ or $\frac{1}{2}$ per cent novocaine sub-peritoneally immediately below the bifurcation of the aorta and the prominence of the sacrum in the middle line.

The Pelvic Viscera.-In addition to the presacral plexus, the sympathetic plexus which accompanies the internal iliac arteries and the ovarian vessels are anaesthetised so that 5-Io c.cs. of solution are injected around these structures separately and extra peritoneally on either side of the brim of the pelvis around the common iliac arteries. The pelvic-ovarian ligament is seen connecting the ovary to the lateral pelvic wall and is likewise saturated with 5 c.cs. of anaesthetic for gynaecological procedures.

In this way the pelvic viscera are insulated and operations can be comfortably performed with the minimum of shock and morbidity.

\section{Local Infiltration of the Viscera}

After the abdominal cavity is explored, the diagnosis established and the required operation, e.g. partial gastrectomy, cholecystectomy, etc., is decided upon, then this organ is infiltrated locally, it corresponds to the direct infiltration of the incision in the abdominal wall.

I. The Stomach.-This is insulated by putting 5-Io c.cs. of anaesthetic around the pyloric artery (right gastric) just above the pylorus. At each end of the greater curvature the left and right gastro-epiploic vessels are likewise injected with Io c.cs. each, the first below the pylorus and the other at the lower pole of the spleen. The left gastric artery is automtaically dealt with when the vagi are treated and later when the stomach is mobilised and turned upwards, when the vessel is visible above the Pancreas.

2. The Gall Bladder.-The gall bladder is seized gently with a sponge forceps and drawn downwards, towards the umbilicus, and then rotated upwards with the liver towards the chest. The stomach is pressed over to the left with a pack and the hepatic flexure of the colon is held down with a pack and a long-bladed retractor and so the gall bladder is exposed. Adhesions between it and the colon and the duodenum are snicked on the gall-bladder surface, thus displaying the neck and the gall bladder bed. The latter is infiltrated with ro c.cs. of anaesthetic. This allows a stronger pull to be exerted and further adhesions between Hartzmann's pouch and the duodenum are divided until the free edge of the gastro-hepatic omentum appears, this contains the common bile duct, the portal vein, and the hepatic artery. The needle is inserted just under the peritoneum of the border of the gastro-hepatic 


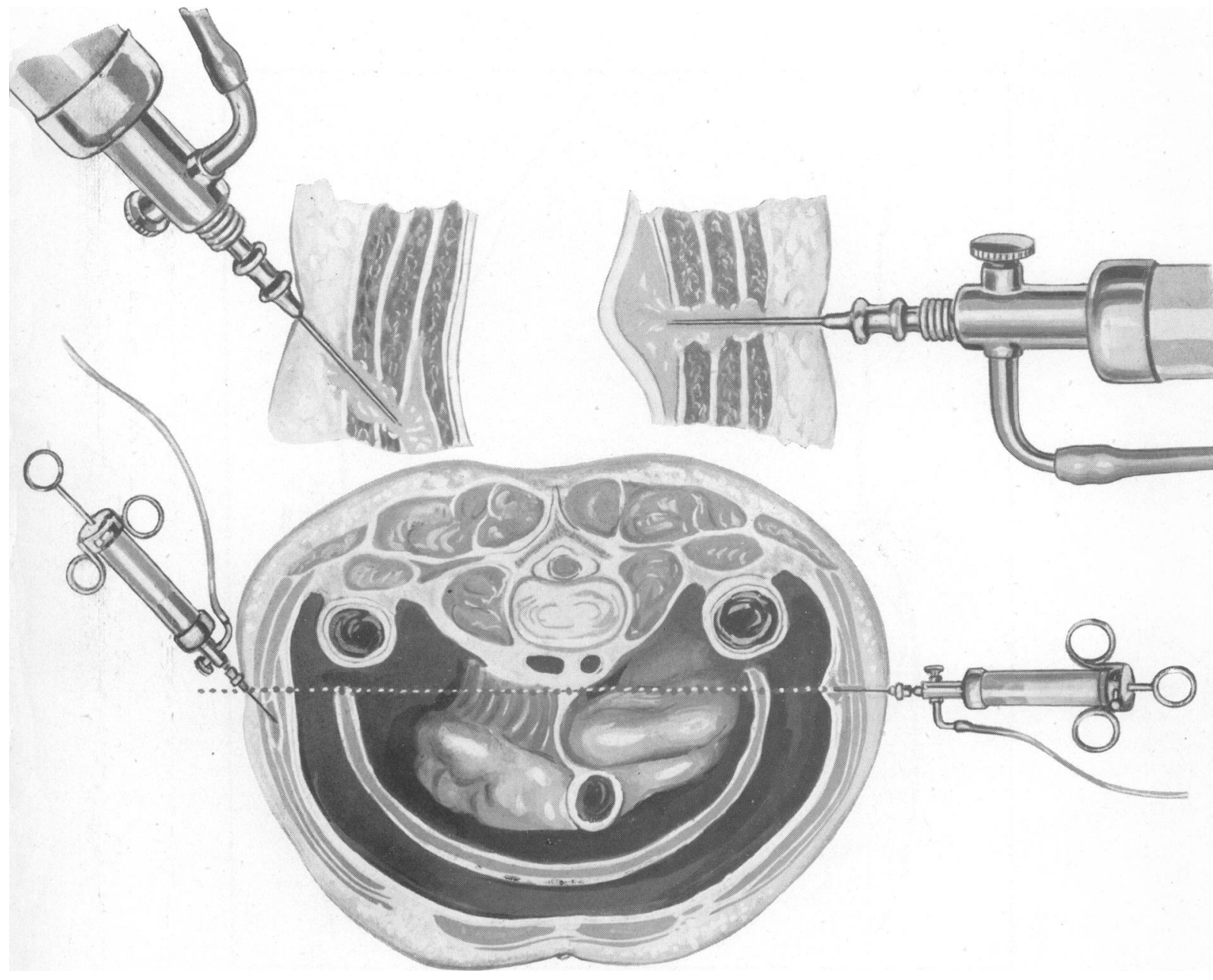

FIG. 3.-Arrow Head Injection. 


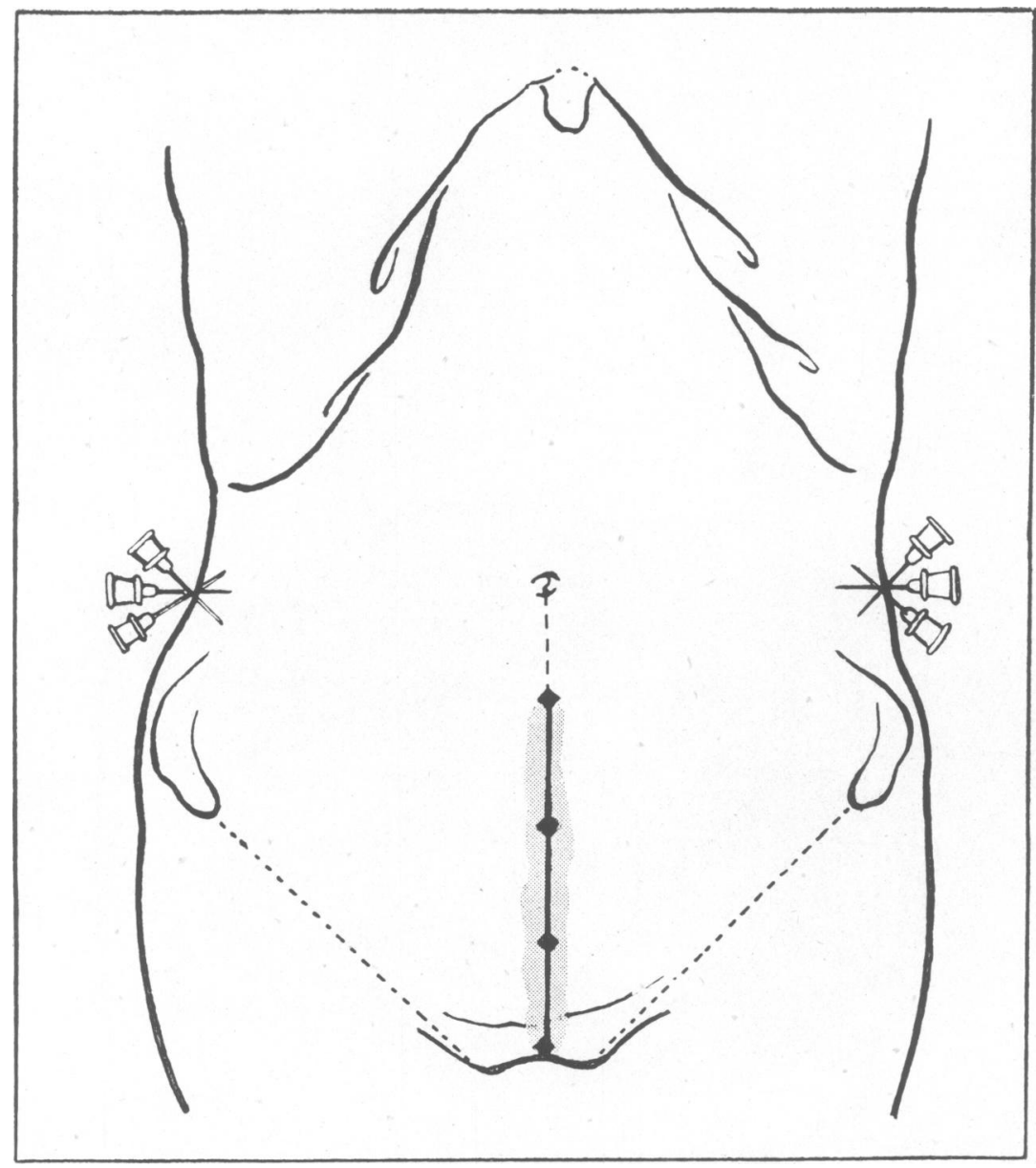

FIG. 4.- Supra pubic cystostomy. 


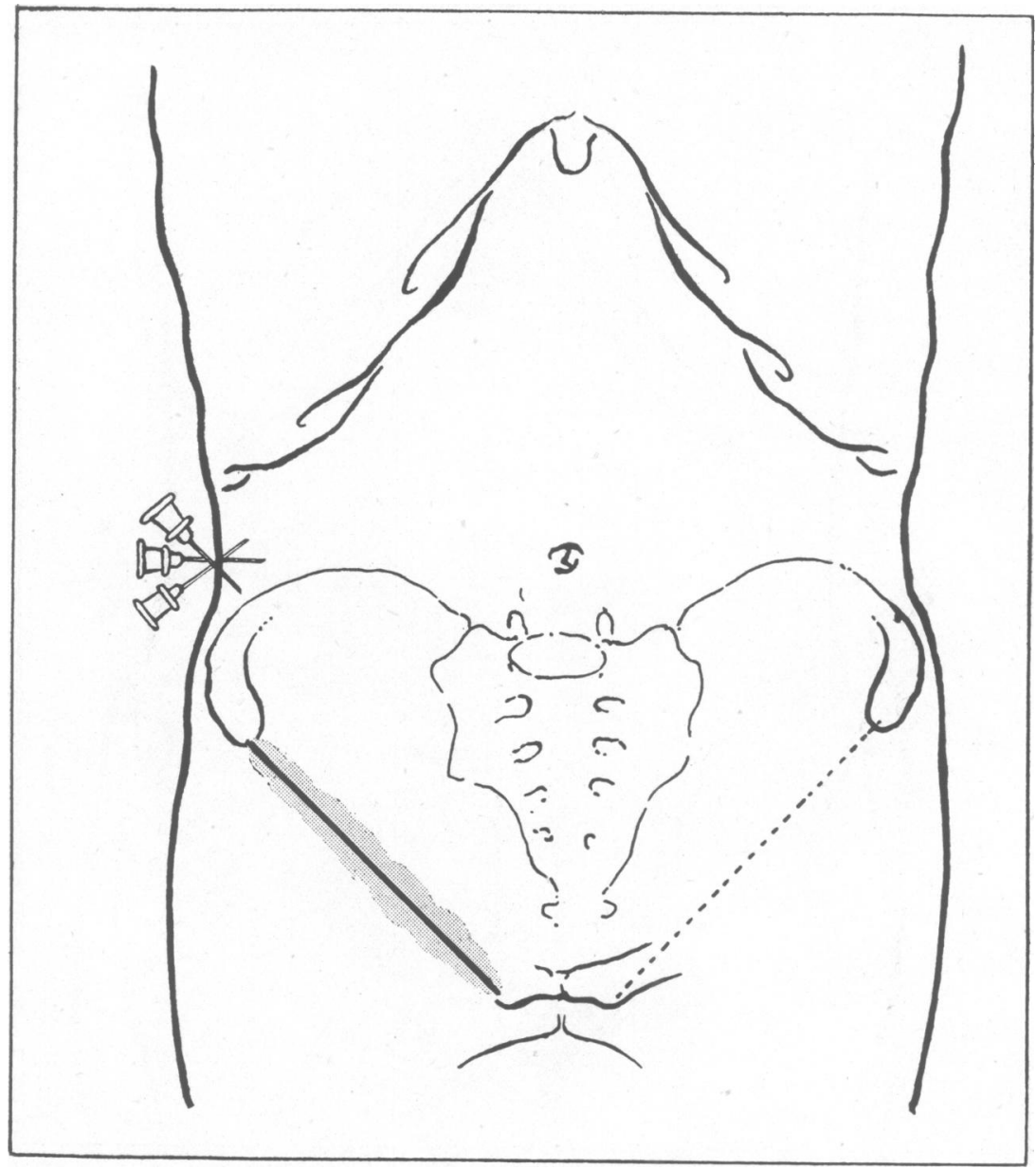

FIg. 5--Inguinal and femoral herniae. 


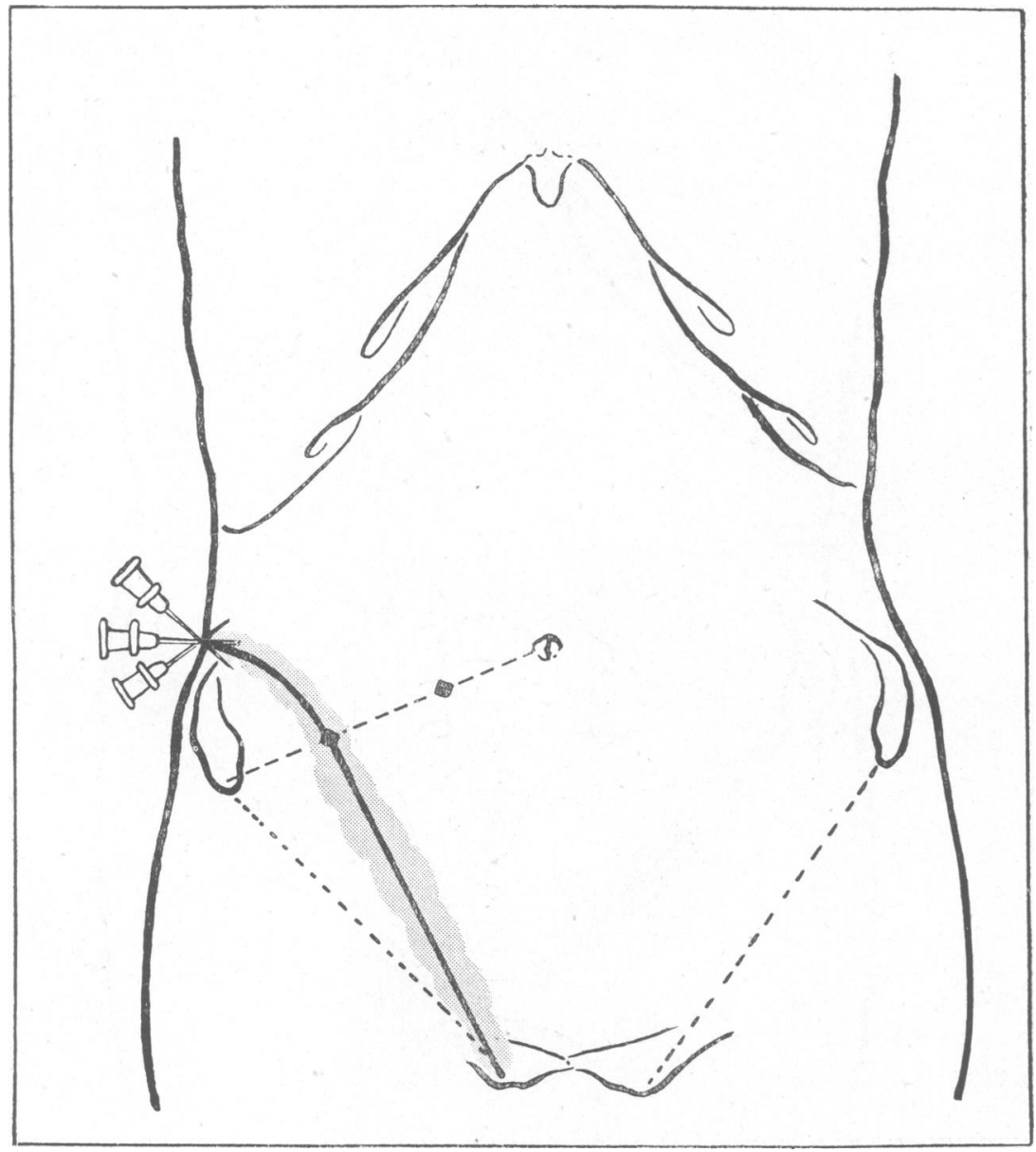

FIG. 6.-Appendix operations on colostomy (left side). 


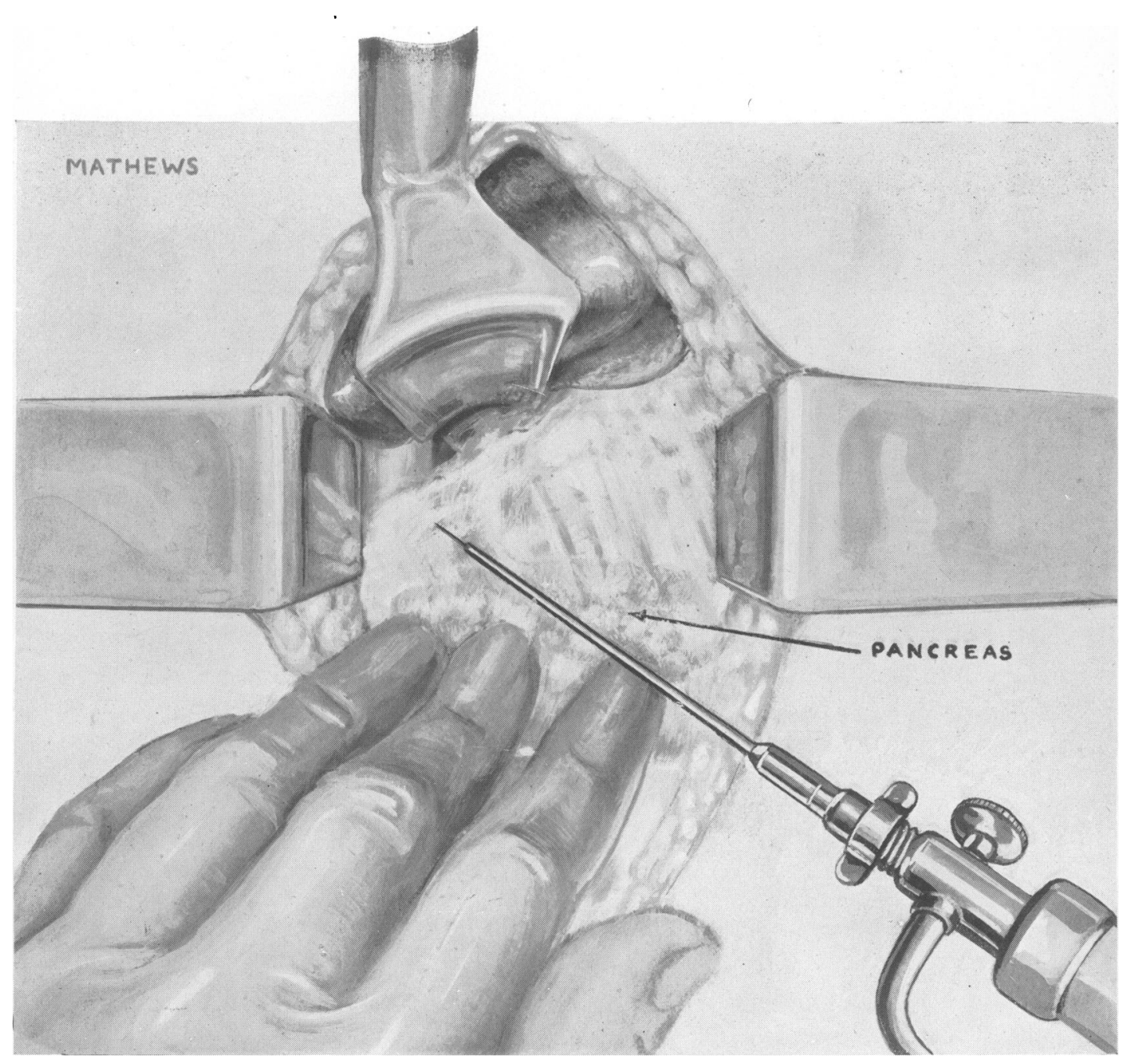

FIG. 7.-Anterior splanchnic injection. 


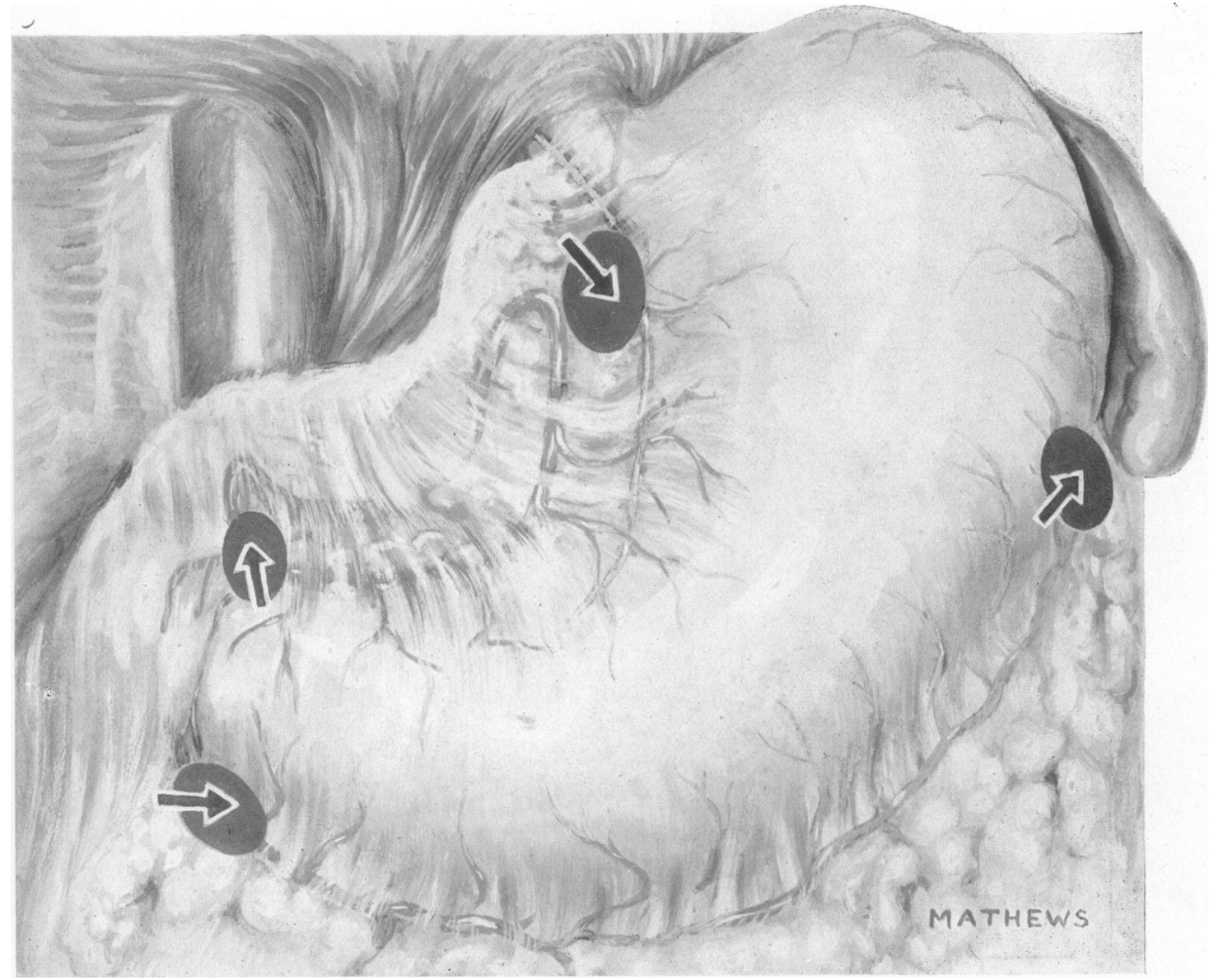

FIg. 8.-Local infiltration of stomach. 


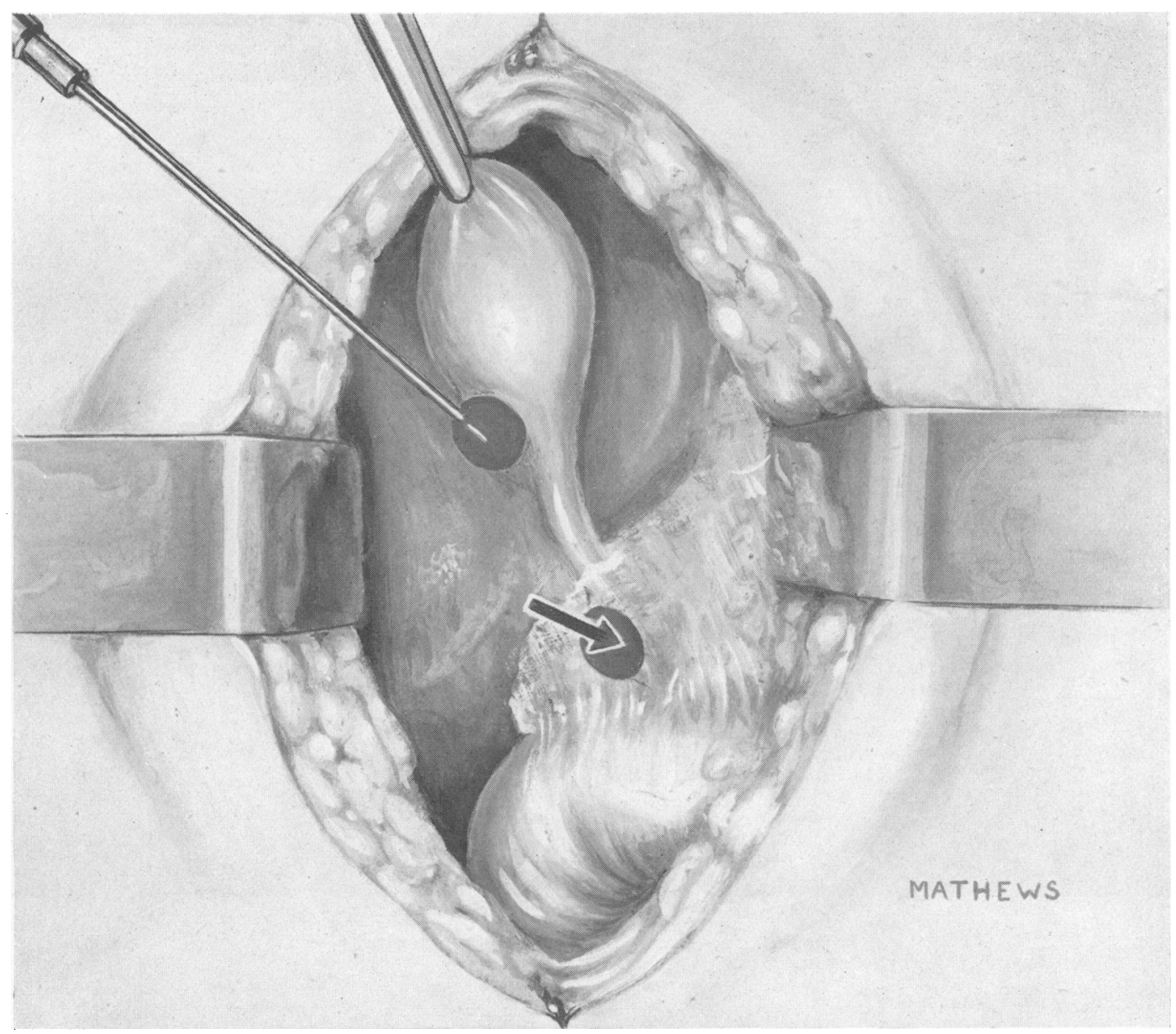

FIG. 9.-Local infiltration for biliary tract operations. 


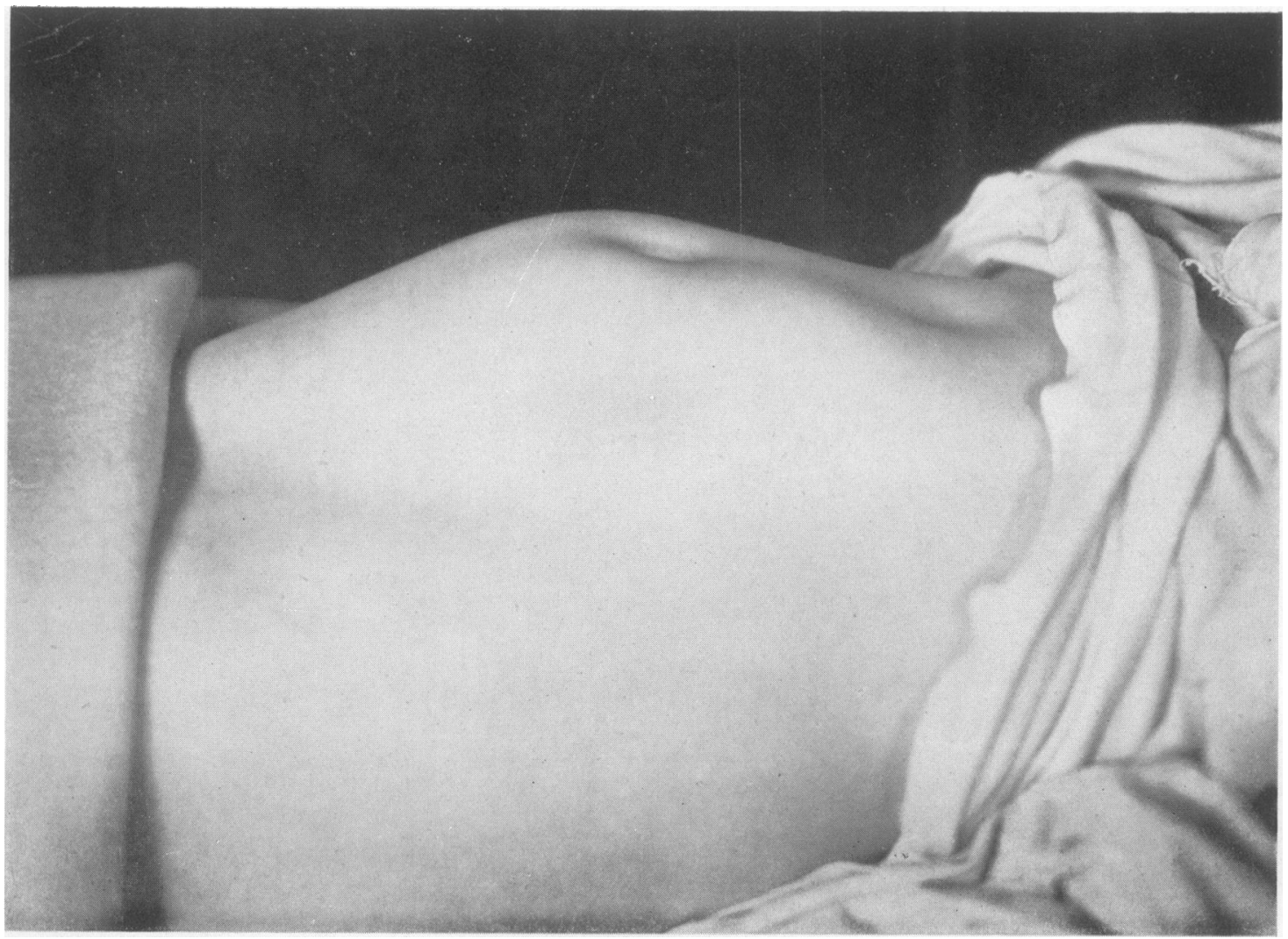

FIG. Io.-Lying down: before operation. 
omentum above the duodenum and the fold is ballooned out with ro c.cs. of anaesthetic which tracks around the common bile duct. The gallbladder and biliary tract are now "damped off" and the operation can be performed confidently and the general anaesthetic can, if it is wished, be discontinued.

3. The Spleen.-The spleen is held forward from the splenic bed by the left hand the lienorenal and gastro splenic ligaments are soaked sub-peritoneally, taking care that the anaesthetic is not inserted into one of the large vessels in the splenic hilum by aspirating first.

\section{Local Anaesthesia for Inguinal Hernia or Femoral Hernia}

Local Infiltration.-The "arrow-head" injection is made on the side of the operation, using Io c.cs. in each direction and taking care to infiltrate the full thickness evenly particularly the external oblique muscle to relax Poupart's ligament, thereby making for a good exposure and accurate work. A taut Poupart's ligament gives poor access and stitches tend to cut out. The incision runs from the anterior superior iliac spine to $\frac{1}{2}$ inch internal to the pubic spine, 3 c.cs. are injected subcuticularly and 3 c.cs. vertically down along this line. At the pubic spine 6 c.cs. are injected towards the scrotum and perineum to desensitise the termination of the long perineal nerves. The deep side of the neck of the hernial sac requires injection when it is exposed; absence of blue, indicates the need.

Femoral Hernia.-In addition to the above insertion, infiltration is needed about the neck of the sac, below Poupart's ligament just external to the pubic spine and especially around the inferior side of the neck. If the Lotheisen approach is used then the neck is infiltrated all round, after incision of the transversalis fascia on the posterior wall of the inguinal canal above Poupart's ligament.

\section{Local Anaesthesia for Supra Pubic Cys- tostomy}

The direct infiltration is of the middle line from the pubis to the umbilicus. The regional block is the "arrow-head" injection on both sides.

Then 20 c.cs. of anaesthetic are pooled supra pubically in the perivesical tissues.

Discussion.-A method of Anaesthesia combining local and general agents for abdominal procedures has been detailed. It has been used since May, I937, and is steadily satisfactory. It minimises strain at operation for the patient and Surgical Team. It is a little slower than other methods. It reduces the post-operative morbidity and nursing. Last, postoperative chests, headaches, retention of urine or abdominal distension are rare and seldom troublesome.

Blood-pressure charts of readings taken during and after the operation show smaller ranges of fall than after procedures under general or spinal anaesthesia.

In my experience no general anaesthetic is consistently satisfactory for any abdominal work, neither upper abdominal nor even herniae. A few patients will not relax, but the local anaesthetic makes this certain.

Balanced anaesthesia is not new, it was first mentioned by Lundy.

It is now inclined to be "old-fashioned" with the advent of tubocurarine which is admittedly superb in competent hands, but it has its dangers. Expert anaesthetists are not everywhere and at all times available, nor is the drug in full supply. Therefore, for the outpost and emergency surgeon, and there are many such, this scheme of Balanced Anaesthesia is invaluable.

Conclusion.-A combination of local and general anaesthesia for abdominal operation is described which has proved successful since May, I937.

\section{PSEUDOCYESIS}

\section{By Mary Doreen Daley, M.D., M.R.C.O.G. \\ (Resident Assistant Obstetric and Gynaecological Surgeon, St. Helier County Hospital, Carshalton, Surrey.)}

Pseudocyesis is a curious condition in which symptoms and signs of pregnancy are simulated sufficiently to convince a woman, her friends and relations, and in many cases her obstetrician, that she is pregnant. The term was first introduced by John Mason Good $(a)$ in I823. Till then false pregnancy was the name most often used, but as Pajot (b) said: "Il n'y a pas de fausses grossesses, il n'y a que faux diagnositics." Other terms fre- quently found in the literature are spurious pregnancy and phantom tumour.

The condition has excited attention from very early times and Hippocrates is said to have observed twelve cases in his practice in 300 B.C. Several famous women in history have been afflicted in this way. Perhaps the most notable example was Mary Tudor who thought that God had not given her a child because she had not 